\title{
РИСКИ И ОСЛОЖНЕНИЯ ЭКО, СПОСОБЫ ИХ ПРОФИЛАКТИКИ И ЛЕЧЕНИЯ
}

\section{RISKS AND COMPLICATIONS OF IVF, METHODS FOR THEIR PREVENTION AND TREATMENT}

\section{O. Shustova}

Summary. The article describes the procedure of in vitro fertilization (IVF), and also analyzes the main risks and complications associated with this procedure. It has been shown that the use of IVF is a significant risk factor for the formation of hypercoagulation, placenta previa, placental ingrowth, premature detachment of a normally located placenta, gestational hypertension and preeclampsia, cholestasis, premature birth, low birth weight, neonatal asphyxia and mortality. The use of IVF is associated with a high frequency of operative delivery, bleeding in the antenatal period and in the third stage of labor, with an increased risk of hysterectomy, pulmonary embolism. Pregnant women after IVF are a high-risk group for early termination of pregnancy.

Improving IVF methods over the past 15-20 years has led to an increase in the frequency of implantation of human embryos, which has led to an increase in the number of multiple pregnancies. Multiple pregnancy is a proven risk factor for the complicated course of pregnancy and childbirth and significantly increases the risk of maternal and perinatal complications. In this regard, the transfer of one embryo has become a priority for treatment with IVF methods.

Unsuccessful IVF attempts are also a risk. The success of IVF programs in women under the age of 35 is much higher than in the late reproductive age. But at present, the protocols of stimulation of superovulation are actively being improved, taking into account the individual (including age) characteristics of patients.

The basic principles of examination, correction and observation of pregnant women are described in the WHO recommendations.

Keywords: infertility, assisted reproductive technologies, in vitro fertilization, IVF, ICSI, IUI, effectiveness, complications, risks, prevention, elimination, pregnancy management.

\author{
Шустова Олеся Вячеславна \\ Д.м.н., Первый Московский государственный \\ медицинский университет имени И. М. Сеченова \\ olesya.olo@icloud.com
}

Аннотация. В статье рассмотрена процедура экстракорпорального оплодотворения (ЭКО), а также проанализированы основные риски и осложнения, связанные с данной процедурой.

Показано, что использование ЭКО является значимым фактором риска формирования гиперкоагуляции, предлежания плаценты, врастания плаценты, преждевременной отслойки нормально расположенной плаценты, гестационной гипертензии и преэклампсии, холестаза, преждевременных родов, маловесного к сроку гестации плода, неонатальной асфиксии и смертности. Применение ЭКО сопряжено с высокой частотой оперативного родоразрешения, кровотечений в антенатальный период и в третьем периоде родов, с повышенным риском гистерэктомии, тромбоэмболии легочной артерии. Беременные после ЭКО относятся к группе высокого риска по прерыванию беременности в ранние сроки.

Совершенствование методов ЭК0 за последние 15-20 лет привело к повышению частоты имплантации эмбрионов человека, что повлекло за собой рост числа многоплодных беременностей. Многоплодная беременность является доказанным фактором риска осложненного течения беременности и родов и значительно повышает риск возникновения материнских и перинатальных осложнений. В связи с этим перенос одного эмбриона стал приоритетной задачей лечения методами ЭКО.

К рискам относят также безуспешные попытки ЭКО. Успешность программ ЭК0 у женщин в возрасте до 35 лет гораздо выше, чем в позднем репродуктивном возрасте. Но в настоящее время активно совершенствуются протоколы стимуляции суперовуляции с учетом индивидуальных (в том числе и возрастных) особенностей пациенток.

Основные принципы обследования, коррекции и наблюдения беременных изложены в рекомендациях В03.

Ключевые слова: бесплодие, вспомогательные репродуктивные технологии, экстракорпоральное оплодотворение, эКО, ИКСИ, ВМИ, эффективность, осложнения, риски, профилактика, устранение, ведение беременности.

При исследовании причин развития бесплодия, было установлено, что в настоящее время причиной бесплодия все чаще является тот факт, что супружеские пары откладывают рождение детей на более поздний период, а на первый план выходит получение образования, карьерный рост, достижение определенных показателей благосостояния. Соответствен- 
но, рождение детей откладывается на окончание репродуктивного периода, что и влечет за собой ряд проблем. В иных случаях, когда бесплодие не связано с возрастом, причиной его развития являются инфекционные патологии, различные заболевания репродуктивной системы мужчины или женщины, наличие абортов, выкидышей, химической контрацепции в анамнезе, неправильный образ жизни, повышенная утомляемость, что влечет за собой нарушение процесса овуляции у женщин, сперматогенеза у мужчин. Стоит отметить, что причиной развития бесплодия может стать как мужской. Так и женский фактор. При этом частота их встречаемости примерно одинакова [4, c.105].

В последнее время обращает на себя внимание тенденция к омоложению бесплодия: резко возросло число молодых пациентов, страдающих бесплодием. Во многом это связано с образом жизни подрастающего поколения: стрессы, вредные привычки, урбанизация, неправильное питание, гиподинамия. Поэтому актуальным является совершенствование имеющихся и поиск новых методов лечения бесплодия [4, с.107].

В последние годы, среди всех методов лечения бесплодия ведущая роль отводится вспомогательным репродуктивным технологиям, в частности, процедуре экстракорпорального оплодотворения (ЭКО). Стоит отметить, что ЭКО проводится двумя основными методами. Классическое ЭКО - это способ, при котором смешивают выделенные яйцеклетки с суспензией сперматозоидов [3; с. 23-25]. Второй способ - ИКСИ, или ICSI, Intra Cellular Sperm Injection - способ оплодотворения, при котором производится инъекция единичного сперматозоида в цитоплазму яйцеклетки. Широкое применение находят различные методы очистки сперматозоидов, например, применяется очистка сперматозоидов путем центрифугирования, что позволяет выделить наиболее активные сперматозоиды, отделить их от плазмы и слизи. Кроме того, специальная очистка оказывает активирующий эффект на сперматозоиды и при легких факторах мужского бесплодия может также решить проблему [4, с. 108-109].

На пятый день оплодотворенная яйцеклетка переносится в полость матки. Предварительно проводится генетическая диагностика. Это обусловлено тем, что перенесенные ранее эмбрионы погибают. Генетическую экспертизу проводят в связи с тем, что примерно 10-15\% беременностей, полученных в результате ЭКО, прерываются в связи с тем, что развитие эмбриона замирает, либо происходит выкидыш. В большинстве случаев, это обусловлено генетическими аномалиями. Однако их можно предотвратить, если своевременно провести генетическую экспертизу [4, с.111].
Одним из наиболее простых методов вспомогательных репродуктивных технологий, является метод ВМИвнутриматочной инсеминации, при котором не требуется извлечения яйцеклетки из организма будущей матери. Выделяют отдельно мужской и женский фактор бесплодия. От того, какой тип бесплодия выявлен у пары, зависит метод дальнейшего лечения [6, с. 600-603].

Несмотря на огромные возможности ЭКО, существуют все же определенные риски и проблемы. Например, многие пары проходят многократные попытки ЭКО, однако имплантации эмбриона при этом не наступает. Причин неудачных попыток ЭКО может быть много. Чаще всего основными причинами выступает тромбофилия, нарушения в системе гемостаза, аутоиммунные процессы. Эти же причины зачастую являются и факторами развития бесплодия [2, с.259]. Процедура ЭКО может быть неудачной в том случае, если у женщины отмечается повышенная склонность к тромбообразованию, нарушена свертываемость крови. Также причиной неудач ЭКО может стать наличие аутоантител. В частности, основная роль в патогенезе отводится антифосфолипидным аутоантителам.

Проблема возникновения осложнений беременности после использования вспомогательных репродуктивных технологий (ВРт), в том числе, ЭКО остается нерешенной до настоящего времени. Определенный интерес с данной точки зрения представляют результаты исследования, проведенного сотрудниками Саратовского государственного медицинского университета [7]. Они приводят данные многоцентровых рандомизированных исследований, выполненных в 2014-2018гг. и посвященных особенностям ведения беременности, наступившей при использовании вспомогательных репродуктивных технологий (экстракорпорального оплодотворения, интрацитоплазматической инъекции сперматозоида), и протоколов ведения беременности (США, Канада, Австралия), наступившей при использовании этих технологий [7].

Использование ЭКО является значимым фактором риска формирования гиперкоагуляции [14], предлежания плаценты [8; 17; 18], врастания плаценты [8; 18], преждевременной отслойки нормально расположенной плаценты [18], гестационной гипертензии и преэклампсии [8; 17], холестаза [11], преждевременных родов $[11 ; 17]$, маловесного к сроку гестации плода [8; 17], неонатальной асфиксии [11], высокой перинатальной смертности $[12 ; 15]$, врожденных пороков развития плода [19], многоплодия [19].

Применение ЭКО сопряжено с высокой частотой оперативного родоразрешения [19], кровотечений в антенатальный период [8; 18] и в третьем периоде родов [9], 
с повышенным риском гистерэктомии [10], тромбоэмболии легочной артерии [16]. Наблюдение за беременностью после ЭКО в связи с высоким риском возникновения вышеуказанной патологии должно осуществляться по следующим направлениям: профилактика преждевременных родов, плацентарных нарушений, гипоксии и асфиксии плода, преэклампсии, своевременная диагностика маловесного плода и возможных пороков его развития, своевременное бережное родоразрешение. Но первый и самый важный принцип наблюдения и родоразрешения - это соблюдение физиологических норм течения беременности и родов [19]. Основные принципы обследования, коррекции и наблюдения беременных изложены в рекомендациях ВОЗ [19; 20] и подтверждены более поздними исследованиями.

Авторами О.В. Яковлевой, Т.Н. Глуховой, И.Е. Рогожиной [7] обоснована целесообразность тщательного мониторинга на протяжении беременности после ВРт, дано патогенетическое обоснование принципов ведения беременности. Подчеркнута важность адекватной прибавки массы тела беременной при многоплодии; с целью своевременной диагностики риска преждевременных родов рекомендована ультразвуковая трансвагинальная цервикометрия в 18-24 неделях беременности, хирургическая коррекция истмико-цервикальной недостаточности до 24 недель гестации или введение акушерского пессария. Показано проведение гормональной поддержки препаратами прогестерона, обоснована целесообразность назначения беременным после ВРт, имеющим риск преэклампсии, низкодозированных препаратов аспирина в сроки 12-16 недель. Представлены данные относительно срока и принципов родоразрешения беременных после использования ВРт [7, с. 140-141].

Беременные после ЭКО относятся к группе высокого риска по прерыванию беременности в ранние сроки. С целью снижения частоты самопроизвольных абортов в первом триместре Российская ассоциация репродукции человека рекомендует продолжать терапию эстрогенами в течение первого триместра беременности при функциональной недостаточности желтого тела или гипоплазии эндометрия. При подтверждении беременности биохимическими показателями, а затем по данным УзИ терапию эстрогенами продолжают с постепенным снижением дозы и отменяют к 12 неделе. Гормональная терапия препаратами прогестерона обычно продолжается до 12-16 недель беременности. С целью своевременной диагностики риска преждевременных родов рекомендуется ультразвуковая трансвагинальная цервикометрия в 18-24 недели беременности [7, с. 141].

У пациенток после ЭКО резко возрастает риск развития преэклампсии. Сама беременность после ЭКО не отнесена отдельно в группу высокого риска по развитию преэклампсии, а имеющиеся данные о более высокой частоте патологии у этого контингента беременных обусловлены возрастом женщин, их экстрагенитальными заболеваниями. Систематическим анализом отмечено, что при преэклампсии имеется значительный дефицит витамина Д, однако его назначение с целью профилактики не привело к снижению частоты этой патологии. Для скрининга на преэклампсию предложено измерять артериальное давление. В то же время наличие протеинурии не может быть использовано как скрининг на преэклампсию, поскольку протеинурия сама по себе не может быть предиктором результатов исхода преэклампсии. Измерение протеинурии используется только для определения степени тяжести преэклампсии. В 2017 году предложен новый, более чувствительный скрининг на риск преэклампсии [O'Gorman N.,] с использованием алгоритма на основе учета сочетания материнских факторов риска, артериального давления, пульсационного индекса маточной артерии в 11-13 недель и содержания фактора роста плаценты в сыворотке крови. он обнаружил 100\% чувствительность (95\% ДИ, 80-100\%) в сроке до 32 недель беременности [13].

Необходимо отметить, что успешность программ ЭКО у женщин в возрасте до 35 лет гораздо выше, чем в позднем репродуктивном возрасте. Но в настоящее время активно совершенствуются протоколы стимуляции суперовуляции с учетом индивидуальных (в том числе и возрастных) особенностей пациенток. Одной из причин обращаемости пациенток позднего репродуктивного возраста по поводу бесплодия, является повторный брак - 42\%. 40\% женщин обращаются по поводу лечения бесплодия в раннем репродуктивном возрасте, однако длительное неэффективное лечение в течение 10 лет и более со сменой ряда лечебных учреждений приводит к тому, что за специализированной помощью эти пациентки обращаются уже в позднем репродуктивном возрасте; отсроченное деторождение по желанию женщины составляет лишь 13\%, гибель единственного ребенка является мотивацией для 5\% женщин [1, с. 28-36].

Репродуктологов это волнует в связи с увеличивающимся потоком обращений в клиники ЭКО пациенток старшей возрастной группы, у которых реализация репродуктивной функции даже с помощью ЭКО является проблемой.

Совершенствование методов ЭКО за последние 15-20 лет привело к повышению частоты имплантации эмбрионов человека, что повлекло за собой рост числа многоплодных беременностей. Многоплодная беременность является доказанным фактором риска ослож- 
ненного течения беременности и родов и значительно повышает риск возникновения материнских и перинатальных осложнений. В связи с этим перенос одного эмбриона стал приоритетной задачей лечения методами ЭКО. Для максимального повышения вероятности наступления беременности в программах ЭКО первостепенное значение имеет возможность выбора эмбриона с наивысшим потенциалом развития. Это позволит сократить время до достижения беременности и облегчит ранжирование оставшихся эмбрионов для криоконсервации, что в последующем будет способствовать переносу потенциально качественного эмбриона в первую очередь [5, с. 47].

Таким образом, основные осложнения связаны с особенностями течения беременности после ЭКО. Ведение беременной после ЭКО заключается прежде всего в тщательном динамическом наблюдении, целевом УЗИ. Следует добиваться адекватной прибавки веса женщиной в каждом триместре беременности, мотивировать выполнение умеренной аэробной нагрузки. С целью своевременной диагностики преждевременного прерывания беременности во втором триместре беременности следует проводить динамическую цервикометрию. Поддержка гестагенами при отсутствии формирования короткой шейки матки у беременных после ВРт ограничивается 16 неделями беременности. Назначение дезагрегантов ограничено только группой высокого риска развития преэклампсии, широкое применение этих препаратов не оправдано. ВРт, возраст женщины более 30 лет, длительность бесплодия не являются самостоятельными показаниями к оперативному родоразрешению. При выборе способа родоразрешения следует опираться прежде всего на оценку состояния здоровья беременной и плода. При родоразрешении через естественные родовые пути необходимо стремиться к максимально щадящим родам [7, с. 143].

\section{ЛИТЕРАТУРА}

1. Гамидов С.И., Овчинников Р. И., Попова А. Ю. Роль мужского фактора бесплодия в программе вспомогательных репродуктивных технологий (обзор литературы) // Андрология и генитальная хирургия.-2017.— Т. 18. — № 18.— С. 28-36.

2. Коган Е.А., Акопова Е. О., Унанян А. Л. Бесплодие при эндометриозе: краткий очерк современных представлений // Древо жизни. — 2017. — № 1(27). C. $251-259$.

3. Неверова Е.Н., Николаева Е. В. Экстракорпоральное оплодотворение - эффективный метод лечения бесплодия // Оренбургский медицинский вестник. - 2014.-Т. 3. - № 1(9).-С. 23-25.

4. Никифоров Д.В., Рябчикова Е.И., Овсянникова Т. В., Попова Ж. Ю. ЭКО: длинная история короткой встречи // Наука из первых рук. - № 2.- 2015.— C. 105-111.

5. Сараева Н.В., Спиридонова Н. В., Тугушев М. Т., Шурыгина О. В., Синицына А. И., Корчагин А. О. Оптимизация переноса одного эмбриона у пациенток с хорошим овариальным резервом // Вестник РГМУ.— 2020. -№ 2.- С. 46-52.

6. Устинов Д. В. Современные методы вспомогательной репродукции в лечении женского и мужского бесплодия // Мир науки, культуры, образования.2014. 一 № 6(49).-C. 600-603.

7. Яковлева 0.В., Глухова Т. Н., Рогожина И. Е. Основные принципы ведения беременности после вспомогательных репродуктивных технологий // Медицинский Вестник Северного Кавказа.— 2020.— № 15(1). - C. 140-145.

8. American College of Obstetricians and Gynecologists. Perinatal risks associated with assisted reproductive technology. Committee 0pinion No. 671. Obstet. Gynecol. 2016; 128: 61-68.

9. Aziz M. M., Guirguis G., Maratto S., Benito C., Forman E. J. Is there an association between assisted reproductive technologies and time and complications of the third stage of labor? Arch. Gynecol. Obstet. 2016;293(6):1193-1196.

10. Cromi A., Candeloro I., Marconi N., Casarin J., Serati M. [et al.]. Risk of peripartum hysterectomy in births after assisted reproductive technology. Fertil. Steril. 2016;106(3):623628.

11. Feng C., Li W. J., He R. H., Sun X.W., Wang G., Wang L. Q. Impacts of different methods of conception on the perinatal outcome of intrahepatic cholestasis of pregnancy in twin pregnancies. Sci. Rep. 2018;8(1):3985. doi.org/10.1038/s41598-018-22387-6

12. Henningsen A. A., Wennerholm U. B., Gissler M., Romundstad L. B., Nygren K. G. [et al.]. Risk of stillbirth and infant deaths after assisted reproductive technology: a nordic study from the CoNARTaS group. Hum. Reprod. 2014;29(5):1090-1096. doi.org/10.1093/humrep/deu031

13. O'Gorman N., Wright D., Poon L.C., Rolnik D. L., Syngelaki A. [et al.] Accuracy of competing-risks model in screening for pre-eclampsia by maternal factors and biomarkers at 11-13 weeks' gestation. Ultrasound Obstet. Gynecol. 2017;49(6):751-755. doi.org/10.1002/uog.17399

14. Orbach-Zinger S., Eidelman L. A., Lutsker A., Oron G., Fisch B., Ben-Haroush A. The effect of in vitro fertilization on coagulation parameters as measured by thromboelastogram. Eur. J. Obstet. Gynecol. Reprod. Biol. 2016; 201:118-120. doi.org/10.1016/j.ejogrb.2016.04.010

15. Sabban H., Zakhari A., Patenaude V., Tulandi T., Aben- haim H. A. Arch Gynecol Obstetrical and perinatal morbidity and mortality among in-vitro fertilization pregnancies: a population-based study. Obstet. 2017;296(1):107-113. doi.org/10.1007/s00404-017-4379-8

16. Sennstrom M., Rova K., Hellgren M., Hjertberg R., Nord E. [et al.]. Thromboembolism and in vitro fertilization — a systematic review. Acta Obstet. Gynecol. Scand. 2017;96(9):1045-1052. doi.org/10.1111/aogs.13147 
17. Society of Obstetricians and Gynaecologists of Canada, Okun N., Sierra S. Pregnancy outcomes after assisted human reproduction. J. Obstet. Gynaecol. Can. 2014;36(1):64-83.

18. Vermey B.G., Buchanan A., Chambers G. M., Kolibiana- kis E.M., Bosdou J. [et al.]. Are singleton pregnancies after assisted reproduction technology (ART) associated with a higher risk of placental anomalies compared with non-ART singleton pregnancies? A systematic review and metaanalysis. BJOG. 2018; 126: 209-218. doi. org/10.1111/1471-0528.15227

19. World Health Organization. Use of multiple micronutrient powders for point-of-use fortification of foods consumed by pregnant women. 2016. Available at: http:// www.who.int/nutrition/publications/micronutrients/guidelines/mmpowders_pregnant_women/en/

20. World Health Organization. WHO recommendations on antenatal care for a positive pregnancy experience. 2016. Available at: http://apps.who.int/iris/bitstre am/10665/250796/1/9789241549912-eng.pdf?ua=1. 15.

(С) Шустова Олеся Вячеславна ( olesya.olo@icloud.com ).

Журнал «Современная наука: актуальные проблемы теории и практики»

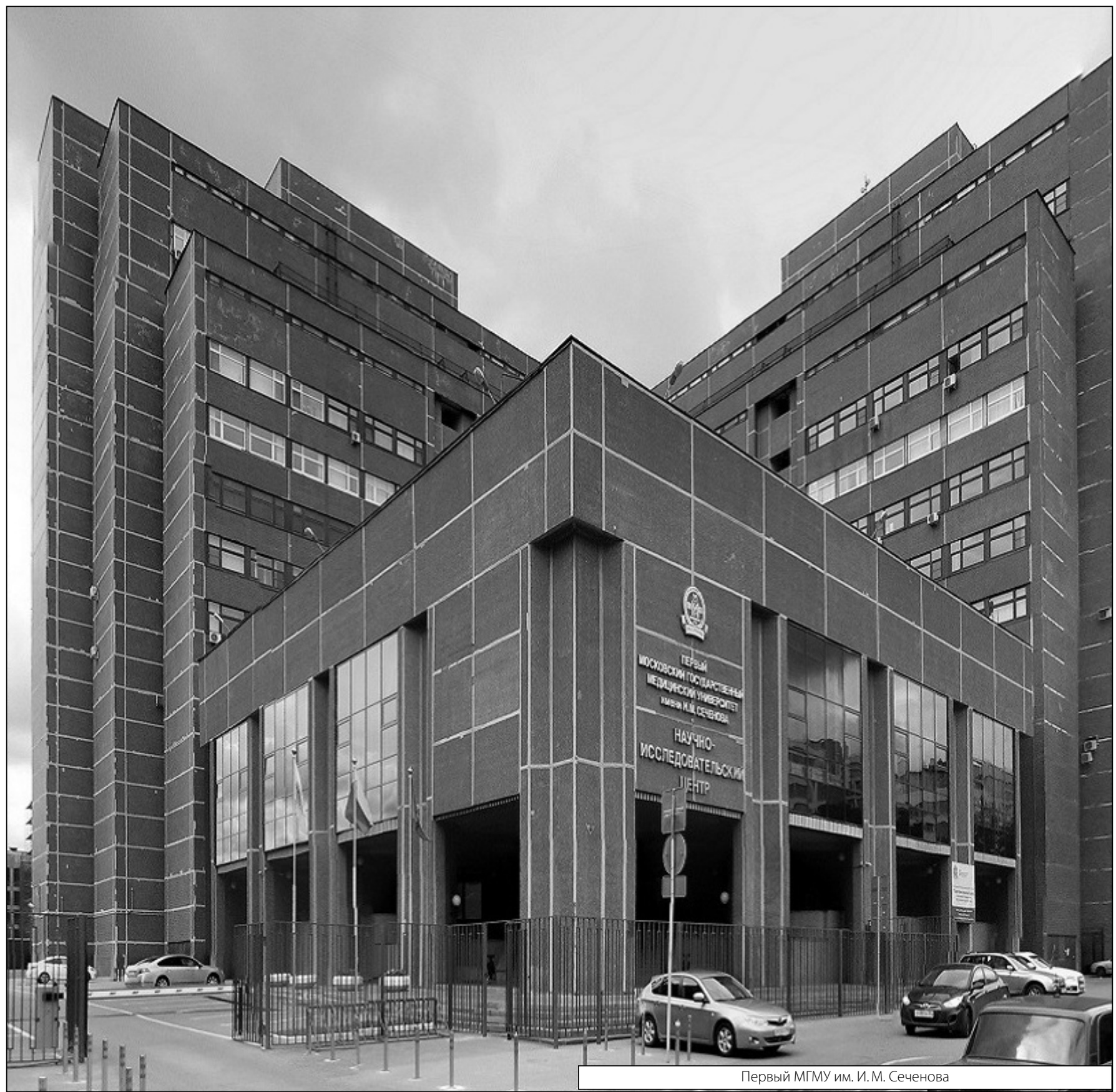

